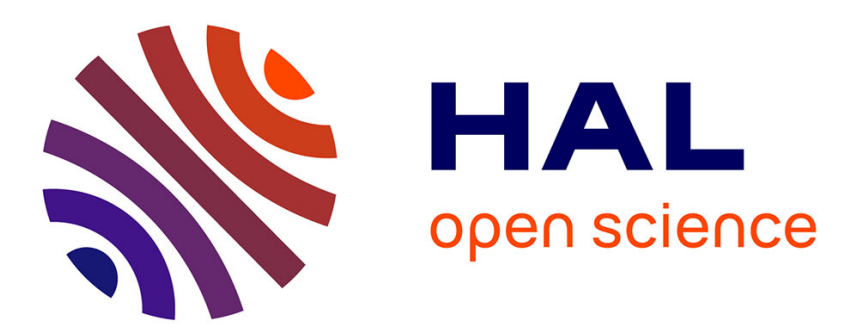

\title{
SAXS investigations of the influence of the length of methylene chain on supermolecular structure of poly(alkylene phosphates)
}

R. Kwiatkowski, A. Wlochowicz

\section{> To cite this version:}

R. Kwiatkowski, A. Wlochowicz. SAXS investigations of the influence of the length of methylene chain on supermolecular structure of poly(alkylene phosphates). Journal de Physique IV Proceedings, 1993, 03 (C8), pp.C8-83-C8-90. 10.1051/jp4:1993817 . jpa-00252248

HAL Id: jpa-00252248

https://hal.science/jpa-00252248

Submitted on 1 Jan 1993

HAL is a multi-disciplinary open access archive for the deposit and dissemination of scientific research documents, whether they are published or not. The documents may come from teaching and research institutions in France or abroad, or from public or private research centers.
L'archive ouverte pluridisciplinaire HAL, est destinée au dépôt et à la diffusion de documents scientifiques de niveau recherche, publiés ou non, émanant des établissements d'enseignement et de recherche français ou étrangers, des laboratoires publics ou privés. 


\title{
SAXS investigations of the influence of the length of methylene chain on supermolecular structure of poly(alkylene phosphates)
}

\section{R. KWIATKOWSKI and A. WEOCHOWICZ}

Textile Institute, Technical University of Łódż, ul. Willowa 2, 43-309 Bielsko-Biała, Poland

\begin{abstract}
The influence of the number of $\mathrm{CH}_{2}$ groups and the evennes of this number on the lamellar arrangement of macromolecules of poly(alkylene phosphates) PPhn were investigated by SAXS method.

The results indicate that deviations from the ideal lamellar model for the polyphosphates with asymmetric mers (n-odd number) are generally larger and qualitatively different as compared with that for the polyphosphates with symmetric mers (n- even number). The heterogeneity of phases were measured by the ratio of the mean square electron density fluctuations calculated from the SAXS curves before and after background subtraction. It was found that the heterogeneity of the PPh8 polyphosphate is smallest. Besides, a linear relationship between the weight average long period $\mathrm{L}_{\mathrm{w}}$ and the molecular mass of the PPhn polyphosphates was confirmed.
\end{abstract}

\section{Introduction.}

In living organisms, NA, TA, RNA acids contain some polymer chains which chemical structure is given by the general formula:<smiles>O=P(O)(O[Te])O[Te]</smiles>

Such a kind of polymers, with large molecular mass, where the $\mathrm{R}$ group is a methyl chain, can be obtained by polycondensation of dimethyl phosphonate with polymethylene diols ${ }^{1,2,3}$. Poly(alkylene phosphates) syntesized in this way, called later as polyphosphates PPhn ( $\mathrm{n}$ is a number of $\mathrm{CH}_{2}$ groups in one mer) have a chemical structure given by the following formula:

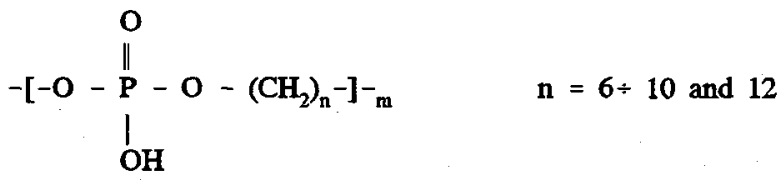

The thermal investigations by means of DSC method ${ }^{3}$ and wide angle X-ray investigations (WAXS) presented in this paper indicate that the polyphosphates are semicrystalline polymers. The results of thermal investigations ${ }^{3}$ show that the melting temperature $T_{m}$ of these polymers nonmonotonically increases with increase in the methyl chain length in a mer. This effect is connected with the evennes of $n$, i.e. PPhn polymers with an odd number of $\mathrm{CH}_{2}$ groups have the lower melting 
temperature than it could be supposed from their location in investigated homologous series of polyphosphates. The symmetry of mers is conected with the evennes of $\mathbf{n}$ number what can be easily shown assuming a trans-type of methyl chain conformation. For an even $n$, the centre of symmetry is located in the middle of the central bond between $\mathrm{CH}_{2}$ groups. So, the $\mathrm{OH}$ groups are uniformly distributed on both sides of the axis of straightened macromolecule. For an odd $\mathbf{n}$ such a symmetry point does not exist and $\mathrm{OH}$ groups are distributed only on one side of the macromolecule axis. This is illustrated by formulae [III] and [IV] related to $n=6$ and $n=7$, respectively. As a result the intermolecular bonds are formed in different conditions which are preferable for an even $n$. Moreover the flexibility of polyphosphates PPhn mers increases with increasing methyl chain length. Both the features, i.e. symmetry and flexibility of mers can significantly effect on the regularity of supermolecular structure and homogeneity of the polyphosphates phases.

\section{I.Experimental.}

\section{II.1.Samples.}

The polyphosphates PPhn were synthesized and characterized in the Center of Molecular and Macromolecular Studies of Polish Academy of Science in Lódź.

The values of the molecular mass $M_{n}$, the glass temperature $T_{8}$, the melting temperature $T_{m}$, the mass of mer $M_{1}$ and the degree of polymerization $\mathrm{m}$ for each sample are collected in tab.1. Besides, this table contains the values of the weight fraction $\mathrm{k}$ of crystalline phase obtained by Hindeleh and Johnson method ${ }^{4,5}$ from our WAXS investigations.

Tab.1. The characterization of polyphosphates samples

a) Results from ref.3

$\mathrm{k}$ - degree of crystallinity (Hindeleh-Johnson method, ref.4,5,6)

\begin{tabular}{||l|c|c|c|c|c|c|c||}
\hline $\begin{array}{l}\text { Symbol } \\
\text { of sample }\end{array}$ & $n$ & $M_{t}$ & $M_{n}$ & $\begin{array}{c}m \\
M_{n} / M_{1}\end{array}$ & $\begin{array}{c}k \\
{[\%]}\end{array}$ & $\begin{array}{c}T_{m}{ }^{a} \\
{\left[{ }^{\circ} \mathrm{C}\right]}\end{array}$ & $\begin{array}{c}T_{8}{ }^{a} \\
{\left[{ }^{\circ} \mathrm{C}\right]}\end{array}$ \\
\hline PPh6 & 6 & 179 & 17400 & 97 & 21 & 68 & $-20 \div-6$ \\
\hline PPh7 & 7 & 193 & 9800 & 51 & 35 & 65 & - \\
\hline PPh8 & 8 & 207 & 8500 & 41 & 43 & 83 & $-11 \div-0.3$ \\
\hline PPh9 & 9 & 221 & 8000 & 36 & 38 & 78 & $-35 \div-31$ \\
\hline PPh10 & 10 & 235 & 10200 & 43 & 30 & 93 & $-8 \div-4$ \\
\hline PPh12 & 12 & 263 & 15200 & 57 & 36 & 102 & $-17 \div-9$ \\
\hline
\end{tabular}

\section{II.2.WAXS investigations.}

The WAXS investigations were performed by means of HZG4 X-ray diffractometer for powdered samples using: a X-ray tube with $\mathrm{Cu}$ target operated at $32.5 \mathrm{kV}$ and $25 \mathrm{~mA}$, a Ni filter, a pulse height analyser and scintillation counter. The WAXS measurements were made employing a step scanning mode with the step of $0.1^{\circ}$ in the range $2^{\circ} \leq 2 \theta \leq 40^{\circ}$.

The WAXS curves of all investigated polyphosphates PPhn have the form typical for semicrystalline polymers. Figures 1.a and 1.b present the WAXS patterns (point lines) obtained for PPh6 and PPh12 samples. The solid lines in these figures present the experimental curves resolved into the diffraction peaks, the amorphous halo and the best fitted theoretical curves. The resolution of WAXS curves was made numerically basing on a modified Hindeleh and Johnson procedure ${ }^{6}$ by means of computer program MINIMAL .

The angular positions and interplanar spacings of the strongest diffraction peaks resolved in this way are collected in tab.2. The weight fraction $\mathbf{k}$ of crystalline phase was determined as the ratio of the total area under resolved crystalline peaks to the total area under unresolved curve.

It is clearly visible from the data presented in tab.2. that the angular position of the first left crystalline peak shifts towards smaller angels $2 \theta$ as the number of $\mathrm{CH}_{2}$ groups increases. The correlation between the interplanar spacings $d_{1}$ related to the angular positions $2 \Theta_{1}$ of that peaks and $n$ is presented in fig.2. The experimental points fulfil the linear relation $d_{1}=a \cdot n+b$ with the slope $a=1.06[\AA ̊]$ and intercept $\mathrm{b}=2.22[\dot{\mathrm{A}}]$ (solid line) obtained by means of the least square method. 


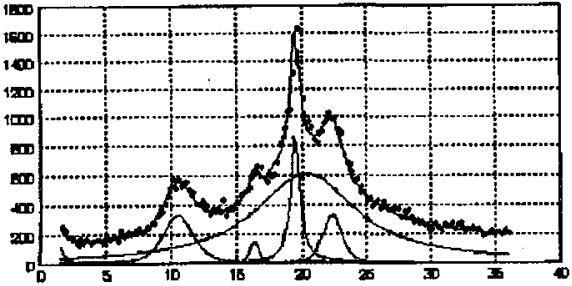

$1 . \mathrm{a}$

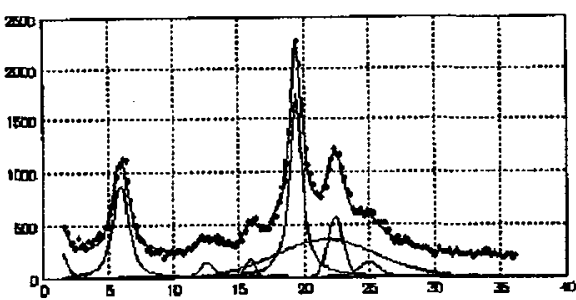

$1 . b$

Fig.1. The WAXS diffraction patterns for PPh6 (1.a) and PPh12 (1.b) samples, resolved into the crystalline peaks and the amorphous halo. Intensity in relative units.

Tab.2. The angular positions and interplanar spacings for the strongest WAXS diffraction peaks $\left(w_{1}\right.$ is the half height width for the first left peaks).

\begin{tabular}{||l|c|c|c|c|c|c||}
\hline Sample & PPh6 & PPh7 & PPh8 & PPh9 & PPh10 & PPh12 \\
\hline $2 \Theta_{1}$ & 10.49 & 9.07 & 8.23 & 7.39 & 6.84 & 5.94 \\
\hline $\mathrm{d}_{1}[\AA ̊]$ & 8.42 & 9.74 & 10.73 & 11.94 & 12.89 & 14.85 \\
\hline $\mathrm{w}_{1}$ & 2.67 & 2.32 & 2.02 & 2.28 & 1.91 & 1.39 \\
\hline $2 \Theta_{4}$ & 19.47 & 19.31 & 19.58 & 19.30 & 19.44 & 19.37 \\
\hline $\mathrm{d}_{4}[\AA ̊]$ & 4.56 & 4.60 & 4.53 & 4.60 & 4.57 & 4.58 \\
\hline $2 \theta_{5}$ & 22.34 & 21.09 & 23.44 & 22.30 & 21.27 & 22.40 \\
\hline $\mathrm{d}_{5}[\AA \AA]$ & 3.98 & 4.21 & 3.79 & 3.98 & 4.18 & 3.97 \\
\hline
\end{tabular}

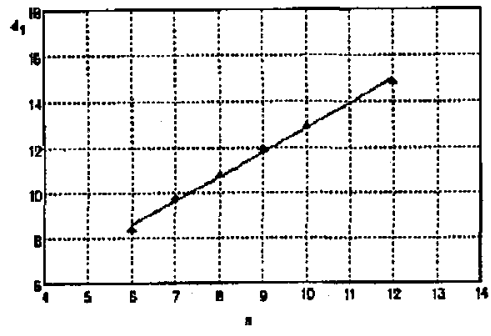

Fig.2 The plot of interplanar spacings $d_{1}$ vs. $n$ ( 1 - the experimental points ).

\section{II.3. SAXS investigations.}

SAXS investigations were carried out by means of Rigaku-Denki diffractometer for powdered samples. A copper target X-ray tube operated at $32.5 \mathrm{kV}$ and $25 \mathrm{~mA}$ was used as the source of radiation. Monochromisation of the beam was achieved using a nickel filter. A scintillation counter was used as a detector.

The SAXS patterns were taken in the range $0.07^{\circ} \div 1.80^{\circ}$ with the step of $0.01^{\circ}$ and in the range $1.80^{\circ} \div 4.25^{\circ}$ with the step of $0.02^{\circ}$. The wieghting function $W_{1}(u)$ of the colimation system was calculated with the method of Hendricks and Schmidt ${ }^{8,9}$ and approximated by a Gaussion function with the standard deviation $p$ of $3.13 \mathrm{~nm}$.

The experimental intensity data were smoothed and corrected for the air scattering, sample absorption, background scattering and collimation distorsions using the computer program FFSAXS5 elaborated by C.G.Vonk ${ }^{10}$.

The scattering curves were normalized to the absolute electron units using the Lupolene plate as a standard (supplied by O.Parr laboratory).

\section{Results and discussion.}

The SAXS diffraction curves of polyphosphates $\mathrm{PPhn}$ with even and odd numbers of $\mathrm{CH}_{2}$ groups in a mer are presented in figures 3.a and 3.b, respectively. The diffraction maxima in these figures are due to X-ray scattering from the lamellar structure of investigated polyphosphates. The examplary one-dimensional scattering functions $I(h) \cdot h^{2}$ vs. $2 \Theta$ related to these diffraction patterns are presented in figures $4 . a$ and $4 . b(h=4 \pi \cdot \sin \theta / \lambda)$. It is seen from fig.3.a and fig.3.b that the maxima for the samples with an odd $n$ are more weak and more broad comparing with those for the samples with an even $\mathbf{n}$. Additionally, the maxima for an even $\mathbf{n}$ differs one from another in their intensities, shapes and angular positions. Moreover, the SAXS curves of the PPhn samples have a different form in the background range $\left(3.0^{\circ} \div 4.25^{\circ}\right.$, see fig.5). These all qualitative differences between the SAXS patters of the PPhn samples will be discused from the point of view of the heterogeneity of crystalline and amorphous domains and from the point of view of the deviations from the ideal lamellar arrangement. 


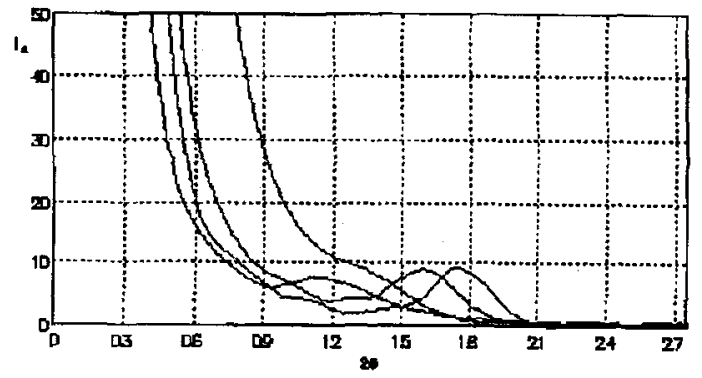

Fig.3.a. The SAXS patterns of PPhn samples for an even $n$. The maxima appear in the following order: PPh6, PPh12, PPh10 and PPh8.( $I_{a}$ - the desmeared intensity in absolute units.)

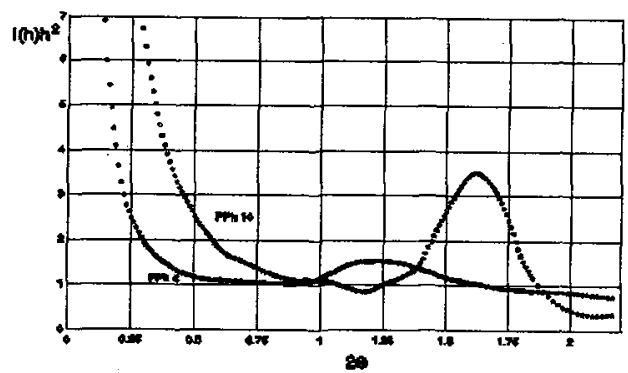

Fig.4.a. The one dimensional scattering functions of PPh6 (the smaller maximum) and $\mathrm{PPh} 10$ (the larger maximum) samples.

\section{III.1. The heterogeneity of phases.}

The background in the small angle X-ray scattering arrises from the heterogeneity of phases and/or wide angle scattering extending towards the small angle region ${ }^{11,12}$. It should be noticed that although the first left wide angle diffraction peak for the $\mathrm{PPh} 12$ sample is much closer to the small angle region $\left(2 \theta_{1}=5.95^{\circ}\right.$;tab.2) than the analogous peak for PPh6 sample $\left(2 \theta_{1}=10.49^{\circ} ;\right.$ tab. 2$)$, the SAXS curve for the first sample goes down up to $4.25^{\circ}$, while for the PPh6 sample the background increases with increasing diffraction angle and for the $\mathrm{PPh}$ 9 sample the background level is constant(see fig.5). Taking it into account the effect of the wide angle scattering on the SAXS curves of polyphosphates PPhn may be omitted and the background may be attributed to the heterogeneity of crystalline and amorphous phases. From the point of view of the phases heterogeneity, polyphosphates can be compared using the factor $f$ given by the expression:

$$
f=\left\langle\eta^{2}\right\rangle_{2} \mid\left\langle\eta^{2}\right\rangle_{1}-1
$$

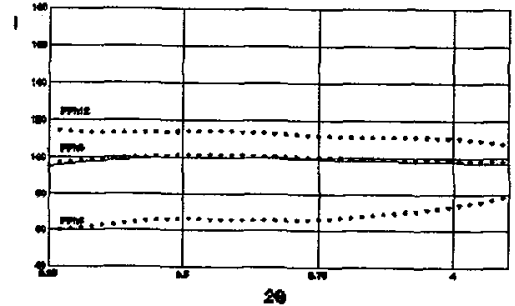

Fig.5 The SAXS curves in the background region for PPh6 (the lowest curve), PPh9 (the intermediate curve) and $\mathrm{PPh} 12$ (the highest curve) samples.

where:

$\left\langle\eta^{2}\right\rangle_{2}$ is the mean square electron density fluctuations calculated directly from smeared SAXS curves $\tilde{\mathrm{I}}_{\text {eq }}(\mathrm{s})$

$(s=2 \cdot \sin \theta / \lambda)$ according to the following equation ${ }^{i 3}$ : 


$$
\left\langle\eta^{2}\right\rangle_{2}=2 \pi \int_{0}^{\infty} \tilde{\mathrm{I}}_{\mathrm{ea}}(\mathrm{s}) \mathrm{s} \mathrm{ds}
$$

and $\left\langle\eta^{2}\right\rangle_{1}$ is the mean square electron density fluctuations calculated from the smeared SAXS curves after background $\tilde{\mathrm{I}}_{\mathrm{B}}(\mathrm{s})$ subtraction:

$$
\left\langle\eta^{2}\right\rangle_{1}=2 \pi \int_{0}^{\infty}\left\{\tilde{I}_{e a}(s)-\tilde{I}_{B}(s)\right\} s d s
$$

For the ideal, homogenous electron density distribution inside the phases the factor $f$ equals to zero and increases with increase in their heterogeneity. The values of the mean square electron density fluctuations $\left\langle\eta^{2}\right\rangle_{2}$, $\left\langle\eta^{2}\right\rangle_{1}$ calculated in the same range of diffraction angles $\left(0.0^{\circ} \div 4.0^{\circ}\right.$ ) and the values of the factor $f$ are collected in tab.3 (the background was subtracted according to the empirical Vonk's approximation ${ }^{13}$ ). The changes of factor $f$ with increasing $\mathbf{n}$ are shown in fig.6. The character of these changes is connected with a different influence of the increasing flexibility of methyl chain in the mer on the homogeneity of polyphosphates phases. An initial increase in the flexibility (i.e. when $n$ increases from 6 to 8 ) causes that a more homogenous structure is formed but for higher $n$ values the thermal fluctuations become more and more important and the heterogeneity of phases increases. In the case of the PPh12 sample, the factor $f$ behaves in different way what is probably connected with different crystalline structure of this polyphosphate as compared with the remaining ones. It is proved by the WAXS investigations (see fig.1.a and 1.b) and a few time higher $\left\langle\eta^{2}\right\rangle_{1}$ value as compared with others (see tab.3).

Tab.3 The values of the mean square electron density fluctuations calculated before $\left(\left\langle\eta^{2}\right\rangle_{2}\right)$ and after $\left(\left\langle\eta^{2}\right\rangle_{1}\right)$ background subtraction and the factor $f$ of the heterogeneity of phases.

\begin{tabular}{||c|c|c|c||}
\hline $\begin{array}{c}\text { Symbol } \\
\text { of sample }\end{array}$ & $\begin{array}{c}\left\langle\eta^{2}\right\rangle_{2} 10^{2} \\
\left(\frac{\mathrm{mol} \mathrm{el} .)^{2}}{\mathrm{~cm}^{6}}\right.\end{array}$ & $\begin{array}{c}\left\langle\eta^{2}\right\rangle_{1} 10^{2} \\
\frac{(\mathrm{mol} \mathrm{el})^{2}}{\mathrm{~cm}^{6}}\end{array}$ & $\mathrm{f}$ \\
\hline PPh6 & 0.261 & 0.139 & 0.88 \\
\hline PPh7 & 0.264 & 0.155 & 0.70 \\
\hline PPh8 & 0.328 & 0.252 & 0.30 \\
\hline PPh9 & 0.431 & 0.314 & 0.37 \\
\hline PPh10 & 0.350 & 0.255 & 0.40 \\
\hline PPh12 & 1.016 & 0.967 & 0.05 \\
\hline
\end{tabular}

\section{III.2 Regularity of the lamellar arrangement.}

The differences between SAXS curves of polyphosphates PPhn corected for the background(see fig.3.a and 3.b) are due to the deviations of polyphosphates structure from the ideal lamellar model, i.e. the presence of the transition layer between phases, the dispersion of crystalline and amorphous layers thicknesses in lamellar stacks and the defects of the second kind ${ }^{13,14,15}$.

The values of the transition layer thickness $E$ were determined by means of the empirical method ${ }^{16}$. The figure 7 shows an example of the experimental plot:

$$
\ln \left[\mathrm{s}^{3} \tilde{I}_{\mathrm{e}}(\mathrm{s}) \exp \left(-\mathrm{p}^{2} \mathrm{~s}^{2}\right)\right] \text { vs. } \mathrm{s}^{1.81}
$$

and the regression line obtained for the PPh10 sample. To determine the $\mathrm{E}$ value, a part of a diffraction curve beyond the second order maximum should be used ${ }^{17}$. Due to relatively high angular positions of diffraction maxima $\left(2 \theta_{\mathrm{m}}=1.75^{\circ}\right.$ for the $\mathrm{PPh} 8$ sample) such a condition can not be fulfilled. Taking it into account and considering large statistical errors of intensities measured at the tail of SAXS curves one may only state that all the polyphosphates have a narrow transition region: $0 \leq E \leq 3[\dot{A}]$ (see tab.4).

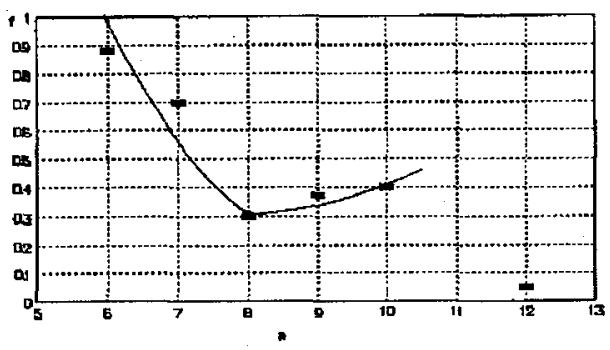

Fig.6. The plot of factor $f$ (eq.1) of PPhn samples vs. $n$. ( - - the experimental points. )

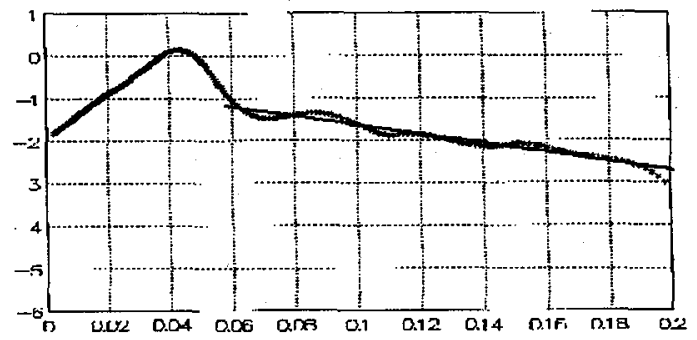

Fig.7 The plot of $\ln \left[\mathrm{s}^{3} \tilde{I}_{\mathrm{e}} \exp \left(-\mathrm{p}^{2} \mathrm{~s}^{2}\right)\right]$ vs. $\mathrm{s}^{1.81}$ and the regresion line obtained for the PPh10 sample. 
The experimental one dimensional correlation function ${ }^{18} \gamma_{1 e}(r)$ obtained from the SAXS patterns (see fig.3.a and 3.b) of PPh6,PPh10 and PPh9 samples (using Vonk's computer program ${ }^{19}$ ) are shown in the fig.8. The function $\gamma_{1 e}(\mathrm{r})$ for the $\mathrm{PPh} 8$ sample has as regular shape as that for the PPh10 sample(fig.8.a). For PPh7 and PPh12 samples the $\gamma_{10}$ (r) functions are similar to that for the PPh9 sample(fig.8.b).

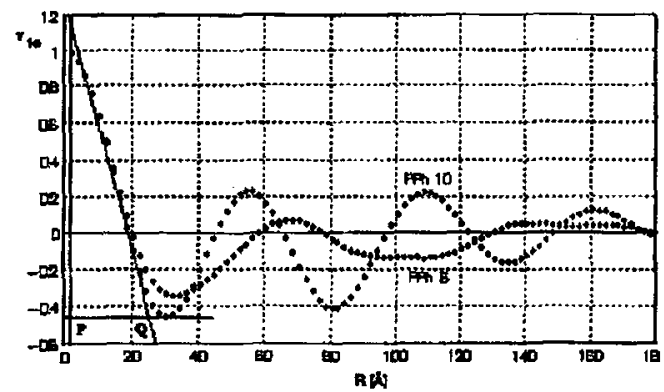

Fig.8.a The experimental one-dimensional correlation functions $\gamma_{1 e}$ for PPh10 ( more regular curve) and PPh6 (less regular curve) samples.

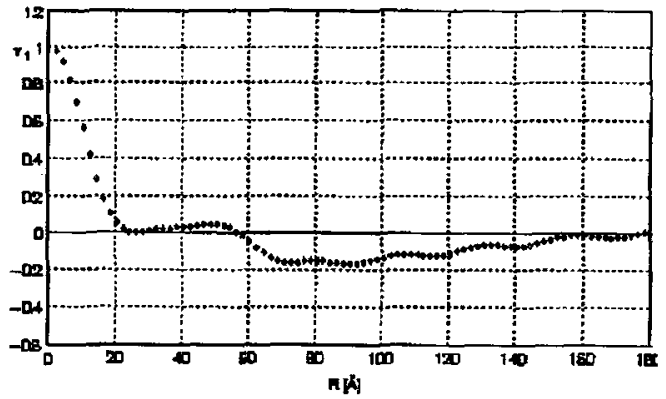

Fig.8.b The experimental one-dimensional correlation function $\gamma_{1 \mathrm{e}}$ for PPh9 sample.

Since, the presence of the transition layer between the phases and the long period dispersion does not destroy the periodicity of the one dimensional correlation function ${ }^{14}$ and because the weight fraction of crystalline phase in the PPhn samples is of the order $20 \div 40 \%$ (tab. 1 ), then the nonperiodic shape of the $\gamma_{1 e}(\mathrm{r})$ functions for $\mathrm{PPh} 7, \mathrm{PPh} 9$ and $\mathrm{PPh} 12$ samples indicates that an undulation and splayings of the lamellar domains (i.e. the second kind defects) ${ }^{15}$ in these polyphosphates exist. On the contrary the form of $\gamma_{1 e}(r)$ functions for the PPhn with an even $\mathbf{n}$ (fig.8.a) indicates on a bigh regularity of lamellar arrangement for the $\mathrm{PPh} 10, \mathrm{PPh} 8$ polyphosphates and on a distinct dispersion of the thicknesses of amorphous and/or crystalline domains ${ }^{14}$ for the PPh6 polyphosphate. The dispersion can be measured by the ratio of the weight average of a long period $L_{w}$, determined form a graph $I(h) \cdot h^{2} v s . h\left(\right.$ or vs.2 2 ), to the number average $L_{a}$ taken from a $\gamma_{1 e}(r)$ function. The values of $L_{p}, L_{n}$ and ratio $L_{w} / L_{n}$ are presented in tab.4. For the PPh8 and PPh10 samples the ratio $L_{w} / L_{n}$ equals to 1 and for the PPh6 sample equals to 1.1 confirming the differences of the regularity of lamellar structure between them.

It should be noticed, that the values of $\mathrm{L}_{\mathrm{w}}$ for PPh6 and PPh12 samples are distinct different from the values of $\mathrm{L}_{\mathrm{w}}$ for the others PPhn samples. It is well known, that the morphology of semicrystalline polymers is considerably dependent on the crystallization temperature and the molecular mass ${ }^{20,21}$. The correlation between the long period $\mathrm{L}_{\mathrm{w}}$ and the molecular mass $M_{a}$ (tab.1) for the PPhn samples is shown in fig.9. If we consider a relatively low accuracy of the determination of $\mathrm{L}_{\mathrm{w}}$ for $\mathrm{PPh} 7$ and PPh9 samples (weak and broad diffraction maxima;see fig.3.b) the correlation between the long period $\mathrm{L}_{\omega}$ and the molecular mass $M_{n}$ presented in fig. 9 can be described through the following linear function: $L=0.0025 \cdot M_{n}+29[\AA]$ (the slope and the intercept have been obtained using the least square method).

From the periodic functions $\gamma_{\mathrm{le}}(\mathrm{r})$ for $\mathrm{PPh} 6, \mathrm{PPh} 8$ and $\mathrm{PPh} 10$ samples one can evalute the volume fraction $\Phi$ of crystalline phase and the thickness of crystalline layers $\left\langle T_{c}\right\rangle^{14,19}$. The intervals within which the volume crystallinity must lie were determinated

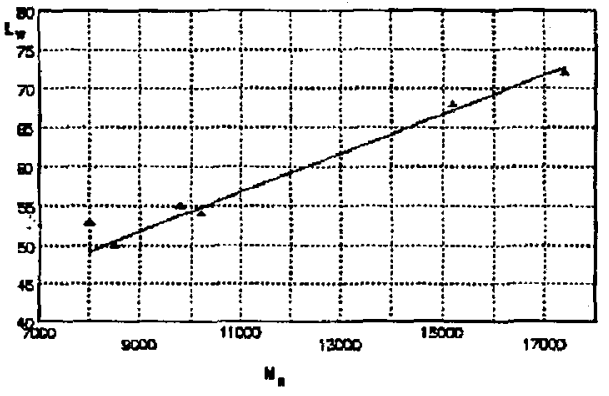

Fig.9 The correlation between the long period $L_{w}$ (tab.4) and the molecular mass $M_{n}$ (tab.1). ( 4 - the experimental points) from the level of the first minimum of $\gamma_{1}(r)$ functions (see fig.8.a) from:

$$
\gamma_{1 \min }=-\phi_{m} /\left(1-\phi_{m}\right) ; \quad \phi_{m} \leq \phi \leq 1-\phi_{m}
$$

The mean thickness $\left\langle T_{c}\right\rangle$ is connected with the sector PQ in fig.8.a by:

$$
P Q=\left\langle T_{c}\right\rangle-\frac{E}{3}(1-\phi)
$$

Because for the all investigated samples the value of $E$ is smaller than $3[\dot{A}]$, hence the second term in eq.5 may be neglected and $\left\langle T_{c}\right\rangle \approx P Q$ (the results are presented in tab.4). The values of $\left\langle T_{c}\right\rangle$ for PPh6 and PPh8 samples are 
closed one to another although the molecular mass of the PPh8 sample is twice smaller from $\mathrm{M}_{n}$ of the PPh6 sample. In tab. 4 the crystallite sizes $t_{1}$ calculated according to Sherrer's equation:

$$
t_{1}=\frac{0.9}{w_{1} \cos \theta_{1}}
$$

from the half height widths $w_{1}$ the angular positions $2 \theta_{1}$ (see tab.2) are shown as well. The relation between $\left\langle\mathrm{T}_{c}\right\rangle$ and $t_{1}$ results from the systematic shifting of the first left WAXS peaks with increasing number of $\mathrm{CH}_{2}$ groups in a mer. There is a satisfying matching between $\left\langle T_{c}\right\rangle$ and $t_{1}$. Taking it into account the values of $t_{1}$ indicates that correlation between the thickness of crystalline layers and molecular mass of the PPhn samples does not exists.

Tab.4 The angular position $2 \theta_{m}$ of the maximum of the $l(h) \cdot h^{2}$ functions and the parameters of the supermolecular structure of the PPhn samples.

\begin{tabular}{|l|l|l|l|l|l|l|l|l|}
\hline $\begin{array}{l}\text { Symbol } \\
\text { of sample }\end{array}$ & $2 \theta_{m}$ & $\begin{array}{l}L_{w} \\
{[\dot{A}]}\end{array}$ & $\begin{array}{l}L_{n} \\
{[\dot{A}]}\end{array}$ & $L_{w} / L_{n}$ & $\begin{array}{l}E \\
{[\dot{A}]}\end{array}$ & $\begin{array}{l}\left\langle T_{c}\right\rangle \\
{[\dot{A}]}\end{array}$ & $\begin{array}{l}t_{i} \\
{[\dot{A}]}\end{array}$ & $\Phi$ \\
\hline PPh6 & 1.23 & 72 & 66 & 1.1 & 1 & 22 & 19 & $0.27 \div 0.73$ \\
\hline PPh7 & 1.60 & 55 & - & - & 2 & - & 22 & - \\
\hline PPh8 & 1.75 & 50 & 50 & 1 & 3 & 24 & 26 & $0.23 \div 0.77$ \\
\hline PPh9 & 1.60 & 53 & - & - & 2 & - & 23 & - \\
\hline PPh10 & 1.62 & 54 & 54 & 1 & 0 & 22 & 27 & $0.12 \div 0.88$ \\
\hline PPh12 & 1.30 & 68 & - & - & 0 & - & 37 & - \\
\hline
\end{tabular}

\section{Conclusions.}

The SAXS investigations of the supermolecular structure of polyphosphates $\mathrm{PPhn}$ with the number $\mathbf{n}$ of $\mathrm{CH}_{2} \mathrm{groups}$ from 6 to 10 and for $n$ of 12 in a mer indicate that the molecular mass $M_{n}$, the evennes of $n$ and the length of methyl chain considerably effect on the lamellar arrangement of their macromolecules.

For the investigated homologous series of polyphosphates the correlation between the long period and the molecular mass fits to a linear function. This result indicates that influence of $M_{n}$ on the long period $L$ is stronger as compared with that for homopolymers ${ }^{2 !}\left(L \sim \sqrt{ } M_{n}\right)$. The difference between these relationships is not yet clearly understood.

The transition layer between the crystalline and amorphous phases of the PPhn polyphosphates is relatively small regardless of methyl chain length in a mer.

The analysis of the experimental one-dimensional correlation functions leads to the following conclusions:

-the asymmetry of mers causes the formation of second kind defects in the lamellar structure (the mers are asymmetrical for an odd $n$; see eq.III and IV)

-the most regular lamellar structure exists in polyphosphates with the mers of the middle flexibility, i.e. for $\mathbf{n}$ equal to 8 and 10

-both large stiffness and high flexibility of mers ( $n$ equal 6 and 12, respectively) do not allow for regular folding of macromolecules as it occurs for the middle flexible mers.

So, the deviations from the ideal lamellar model are larger for polyphosphates with an odd $\mathbf{n}$ as compared with that for polyphosphates with an even $n$.

The changes of the ratio of the mean square electron density fluctuations calculated from the SAXS curves before and after the background subtraction indicate that for $n$ varying from 6 to 10 the most homogenous phases are formed for $n$ equals to eight. For this number of $\mathrm{CH}_{2}$ groups in a mer the flexibility of the mer is not too high, hence thermal fluctuations are relatively small.

Acknowledgments.

Authors would like to thank J.Pretula for the sythesis and characterisation of the PPhn samples. 


\section{References.}

1. J.Pretula, S.Penczek, Macromol.Chem. Rapid Commun.9, 731 (1988)

2. J.Pretula, S.Penczek, Macromol. Chem. 191, 671 (1990)

3. J.Pretula, S.Penczek, Macromol., in the press (1993)

4. A.M.Hindeleh, D.J.Johnson, J.Phys.D 4, 259 (1971)

5. A.M.Hindeleb, D.J.Johnson, Polymer 19, 27 (1978)

6. S.Rabiej, Eur.Polym.J. 27, 9 (1991)

7. S.Rabiej, A.Wlochowicz, J.Appl.Polym.Scj. 46, 1205 (1992)

8. R.W.Hendricks, P.W.Schmidt, Acta Phys. Austriaca 26, 97 (1967)

9. R.W.Hendricks, P.W.Schmidt, Acta Phys.Austriaca 37, 20 (1973)

10. C.G.Vonk, J.Appl.Cryst. 8, 340 (1975)

11. J.Rathje, W.Ruland, Colloid Polym.Sci. 254, 358 (1976)

12. W.Wiegand, W.Ruland, Prog.Colloid Polym.Sci. 66, 355 (1979)

13. C.G.Vonk, J.Appl.Cryst. 6, 81 (1973)

14. C.G. Vonk, G.Kortleve, Kolloid Z.Z. Polymer 220, 19 (1967)

15. C.G.Vonk, J.Appl.Cryst. 11, 540 (1978)

16. J.T.Koberstein, B.Mora, R.S.Stein, J.Appl.Cryst. 13, 34 (1980)

17. W.Ruland, Collid Polymer Sci. 255(5), 417 (1977)

18. A.Guinier, G.Furnet, Small Angle Scattering of X-Rays, Wiley New York, 111-120 (1955)

19. C.G.Vonk, A.J.Pijpers, J.Polym.Sci. 23, 2517 (1985)

20. L.Mandelkern, Faraday Discuss. Chem.Soc. 68, 310 (1979)

21. M.Glotin, L.Mandelkern, Colloid Polym.Sci. 260, 182 (1982)

22. E.Robelin-Souffache, J.Rault, Macromol., 22, 3581 (1989) 